\title{
Is Omicron Less Severe than Delta?
}

\author{
Mini Review
}

Volume 3 Issue 2- 2022

\author{
Author Details \\ Zuber Ahmad *, Ishma Zubair ${ }^{2}$, Nibras Zuber \\ ${ }^{1}$ Department of Resp Medicine, J N Medical College, India \\ ${ }^{2}$ Asian Institute of Medical Sciences, India \\ *Corresponding author \\ Zuber Ahmad, Department of Resp Medicine, J N Medical College, AMU, Aligarh, India \\ Article History \\ Received: January 23, 2022 Accepted: January 24, 2022 Published: February 02, 2022
}

\section{Introduction}

As world braces for the peak of a new Covid wave with Omicron taking over all other existing variants, scientists are scrambling to find how this heavily-mutated virus spreads so quickly and affects people at an alarmingly high rate. The surge in the cases of omicron variants has raised questions on the effectiveness of the vaccines being administered. The symptoms of this variant are milder compared to the Delta variant. But due to the high rate of transmissibility, it has become the dominant variant across the globe. The early doubling time of the Omicron variants was calculated to be about 1.2 days, as compared to 1.7 and 1.5 days of Beta and Delta respectively [1]. These data indicate that the Omicron variant is more infectious than the Delta and Beta variants.

The Omicron variant (B.1.1.529) is a variant of SARS-CoV-2 that was first reported to the World Health Organization (WHO) from South Africa on 24 November 2021 [2,3] On 26 November, the WHO's Technical Advisory Group on SARS-CoV-2 Virus Evolution declared B.1.1.529 a fifth variant of concern (VOC) and designated it with the Greek letter omicron [2]. The previous four VOCs were Alpha (B.1.1.7), Beta (B.1.351), Gamma (P.1), and Delta (B.1.617.2). They all resulted in a new wave of pandemic and thousands of deaths across the whole world [4].

\section{Mutations}

Analysis of the genomic sequences of the Omicron variant has revealed a large number of non-synonymous mutations, including several ones in spike that have been proved to be involved in transmissibility, disease severity, and immune escape. Overall, more than 60 substitutions/deletions/insertions have been identified in the Omicron variant [5] making Omicron a variant possessing the largest number of mutation sites of all SARSCoV-2 variants characterized so far. The mutations in omicron variant comprise 50 non synonymous mutations, 8 synonymous mutations, and 2 non-coding mutations [6]. Thirty-two mutations affect the spike protein, the main antigenic target of antibodies generated by infections and of many vaccines widely administered. Many of these mutations had not been observed in other strains $[7,8]$.

\section{Possible Consequences}

Omicron multiplies around 70 times faster than the Delta variant in the bronchi but evidence suggests it is less severe than previous strains, especially compared to the Delta variant $[9,10]$. Omicron is less able to penetrate deep lung tissue [11]. Omicron infections are 91 percent less fatal than the delta variant, with 51 percent less risk of hospitalization [12]. Overall, the extremely high rate of spread, combined with its ability to evade both double vaccination and the body's immune system, means the total number of patients requiring hospital care at any given time is still of great concern [11].

\section{The Difference in the Symptoms of Omicron and}

\section{Delta}

Delta and Omicron both are mutant variants of the original strain of the COVID-19, which originated in China in 2019. Delta was first identified in India in 2020 and later it led to a wave of the coronavirus in the world claiming millions of lives. The symptoms of both variants are different from each other. Experts reveal that fatigue, joint pain, cold and headaches are four common signs of omicron that are different from that of the Delta variant. Apart from these, loss of smell and taste, which were common signs of Delta, are hardly seen in the case of the Omicron [13].

\section{Omicron does not Lead to Breathlessness}

Omicron does not lead to breathlessness like Delta or another previous variant of the COVID-19 because the new variant multiplies in the throat instead of the respiratory system [14-16] and the impact of omicron infection is likely to be minimal on the lungs. Unlike Delta infection in which a large group of the population was hospitalized 
and suffered from pneumonia, the same is not happening in the case of the new variant.

\section{The Effect of Natural Immunity and Vaccine on Omicron}

Omicron variant hosts an unprecedented number of mutations in its spike gene and early reports have provided evidence for extensive immune escape and reduced vaccine effectiveness [17-20]. The risk of breakthrough infection is higher in both cases. A recently published study demonstrates that neutralizing activity of sera from convalescent and double vaccinated participants was undetectable to very low against B.1.1.529 while neutralizing activity of sera from individuals who had been exposed to spike three or four times was maintained, albeit at significantly reduced levels [21]. The study demonstrates that Omicron drastically escapes vaccine-induced immunity after primary vaccination and exhibits increased infectivity in vitro, raising the potential for increased transmissibility.

All variants of Covid-19, including the Delta variant that is dominant worldwide, can cause severe disease or death, in particular for the most vulnerable people, and thus prevention is always a key. The most effective steps individuals can take to reduce the spread of the Covid-19 virus is to keep a physical distance of at least 2 yards from others, wear a well-fitting mask, open windows to improve ventilation, avoid poorly ventilated or crowded spaces, keep hands clean, cough or sneeze into a bent elbow or tissue, and get vaccinated when it's their turn

\section{References}

1. Karim SSA, Karim QA (2021) Omicron SARS-CoV-2 variant: a new chapter in the COVID-19 pandemic. Lancet 398(10317): 2126-2128.

2. (2021) Classification of Omicron (B.1.1.529): SARS-CoV-2 Variant of Concern. World Health Organization.

3. Gowrisankar A, Priyanka TM, Banerjee S (2022) Omicron: a mysterious variant of concern. The European Physical Journal Plus 137(1): 100.

4. WHO (2021) Tracking SARS-CoV-2 variants.

5. GISAID (2021) Covariants.

6. Haseltine WA (2021) Omicron Origins. Forbes.

7. Cookson C, Barnes O (2021) What we know about Omicron variant that has sparked global alarm. Financial Times.
8. Callaway E (2021) Heavily mutated Omicron variant puts scientists on alert. Nature 600(7887): 21

9. Harvard Medical School (2022) Coronavirus Resource Center - Harvard Health. Harvard Health Publishing.

10. David Leonhardt (2022) Omicron Is Milder. The New York Times.

11. (2021) Lung tissue study sheds light on fast Omicron spread. CIDRAP.

12. Lewnard JA, Hong VX, Patel MM, Kahn R, Lipsitch M, et al. (2022) Clinical outcomes among patients infected with Omicron (B.1.1.529) SARS-CoV-2 variant in southern California.

13. https://www.gavi.org/vaccineswork/top-5-symptoms-be-awareomicron

14. Mc Mahan K, Giffin V, Tostanoski LH, Chung B, Siamatu M, et al. (2022) Reduced Pathogenicity of the SARS-CoV-2 Omicron Variant in Hamsters. bioRxiv.

15. Peacock TP, Brown JC, Zhou J, Thakur N, Newman J, et al. (2022) The SARS-CoV-2 variant, Omicron, shows rapid replication in human primary nasal epithelial cultures and efficiently uses the endosomal route of entry. bioRxiv.

16. Meng B, Ferreira IATM, Abdullahi A, Goonawardane N, Saito A, et al. (2021) SARS-CoV-2 Omicron spike mediated immune escape and tropism shift. bioRxiv.

17. Cele S, Jackson L, Khoury DS, Khadija Khan, Moyo-Gwete T, et al. (2021) SARS-CoV-2 Omicron has extensive but incomplete escape of Pfizer BNT162b2 elicited neutralization and requires ACE2 for infection. medRxiv.

18. Andrews N, Stowe J, Kirsebom F, Toffa S, Rickeard T, et al. (2021) Effectiveness of COVID-19 vaccines against the Omicron (B.1.1.529) variant of concern. medRxiv.

19. Wilhelm A, Widera M, Grikscheit K, Toptan T, Schenk B, et al. (2021) Reduced Neutralization of SARS-CoV-2 Omicron Variant by Vaccine Sera and Monoclonal Antibodies. medRxiv.

20. Rössler A, Riepler L, Bante D, Laer DV, Kimpel J (2021) SARS-CoV-2 B.1.1.529 variant (Omicron) evades neutralization by sera from vaccinated and convalescent individuals. medRxiv.

21. Carreño JM, Alshammary H, Tcheou J, Singh G, Raskin A, et al. (2021) Activity of convalescent and vaccine serum against SARS- Cov-2 omicron. Nature. 